\title{
Studying the Underlying Event in $p+p$ collisions at the LHC
}

\author{
A. G. Agócs* \\ Eötvös University, \\ 1/A Pázmány P. stny., Budapest 1117, Hungary \\ MTA KFKI RMKI, \\ 29-33 Konkoly-Thege M. út, Budapest 1121, Hungary \\ E-mail: agocs@rmki.kfki.hu

\section{P. Lévai} \\ MTA KFKI RMKI, \\ 29-33 Konkoly-Thege M. út, Budapest 1121, Hungary \\ E-mail: plevai@rmki.kfki.hu
}

\begin{abstract}
In proton-proton collisions at $\mathrm{LHC}$ energies $\left(\sqrt{s_{N N}}=14 \mathrm{TeV}\right)$ the expected charged particle multiplicity could be as high as in $\mathrm{Cu}+\mathrm{Cu}$ collisions at RHIC energies $\left(\sqrt{s_{N N}}=200 \mathrm{GeV}\right)$. Since collective phenomena have been observed in heavy ion collisions, then similar effects may appear in high multiplicity $\mathrm{p}+\mathrm{p}$ collisions at LHC. For detailed investigation we consider the properties of the underlying event (UE) which includes particles outside the jets. We improved the original jet-UE separation method of the CDF Collaboration and studied it in simulated $\mathrm{p}+\mathrm{p}$ collisions.
\end{abstract}

European Physical Society Europhysics Conference on High Energy Physics, EPS-HEP 2009,

July 16 - 222009

Krakow, Poland

* Speaker. 
At the Relativistic Heavy Ion Collider (RHIC) proton-proton, proton-nucleus and nucleusnucleus collisions have been performed up to $\sqrt{s_{N N}}=200 \mathrm{GeV}$ energy. The $\mathrm{p}+\mathrm{p}$ collisions were considered as elementary processes, as reference events. The appearance of collective phenomena was intensively studied in heavy ion collisions where high particle multiplicity could have been observed jet energy loss and elliptic flow effects have been discovered. At LHC energies, where $\sqrt{s_{N N}}=7 \mathrm{TeV}$ will be reached in 2010 , and $14 \mathrm{TeV}$ later we expect large entropy production, and events with as high multiplicities as in mid-central $\mathrm{Cu}+\mathrm{Cu}$ collisions at RHIC energies. Thus we may expect the appearance of collective phenomena in high multiplicity $p+p$ collisions. The study of these events demands the separation of the jets and their jet cones from the rest of the event called the underlying event (UE).

At FERMILAB energies $\left(\sqrt{s_{N N}}=1.96 \mathrm{TeV}\right)$ this separation has been performed and investigated in details by the CDF Collaboration [1]. The jets are defined by a jet finder algorithm, usually considering high energy particles in a cone-like area. There are multiple algorithms in use: simple cone, $k_{\mathrm{T}}$, anti- $k_{\mathrm{T}}$, SISCONE [2,3]. Previous studies have defined the UE in terms of a geometry fixed by the jet with the highest energy, see left panel of Fig. 1. These efforts were directed to characterise the UE as a background and subtract it from the signal. The original method did not involve any jet finding algorithm, tracks with the highest $p_{\mathrm{T}}$ were used, simply. The main shortcomings of this definition is the small "transverse region" (the area which most sensitive to the UE component of the Monte Carlo event generators), and the independence on the pseudo-rapidity.

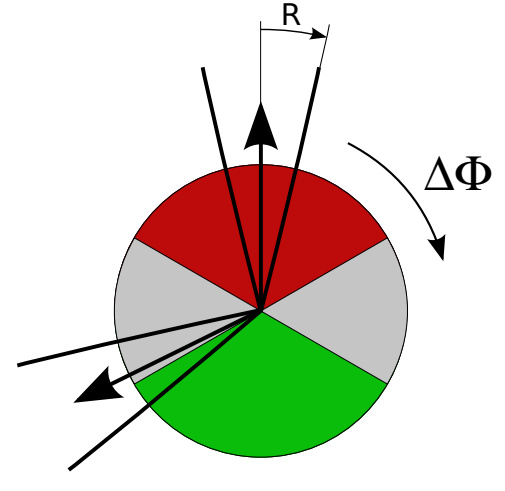

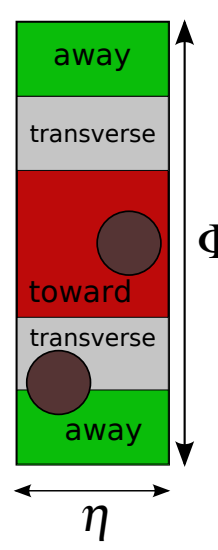
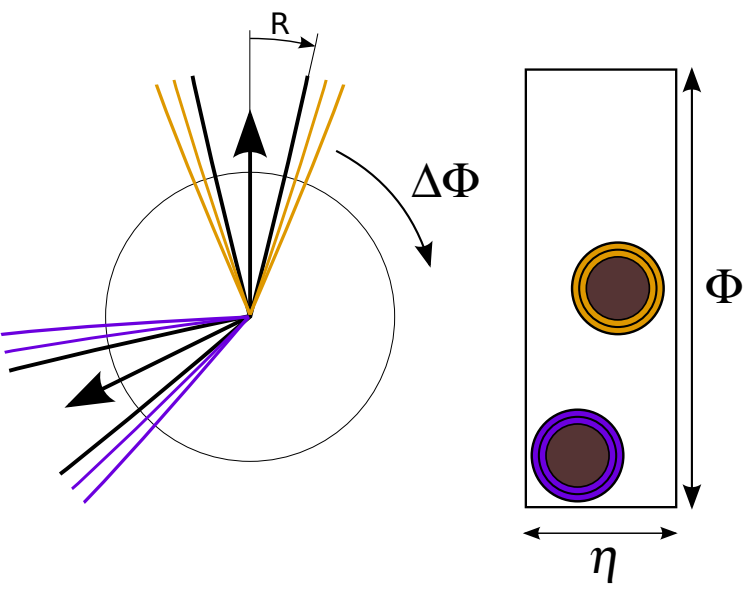

Figure 1: (Colour online.) Left panel: the fixed geometry of the CDF definition [1] and a non back-to-back dijet event. The arrows represent the centre of the jets and the shaded areas represent the regions as defined by CDF. Right panel: the surrounding cones (part of the UE) around the jet cone in the transverse plane and the $\Phi-\eta$ plane.

We are introducing an improved definition of the UE [4], based on modern jet finding algorithms and taking into account that these jets are confined along the $\eta$ axis as well. In this approach the described region is extended compared to the "transverse" region defined by the CDF Collaboration. The UE is defined as the whole region that is outside of the jet cones and in the acceptance region. This implies that a cone-based jet finding algorithm is used. Using a cone-based algorithm also has the advantage that we can define subregions of the UE, called "surrounding cones", in computationally feasible way. The surrounding cones are cylindric areas around the cones of the 
mul: $50-60, \max : 3 \mathrm{GeV}$

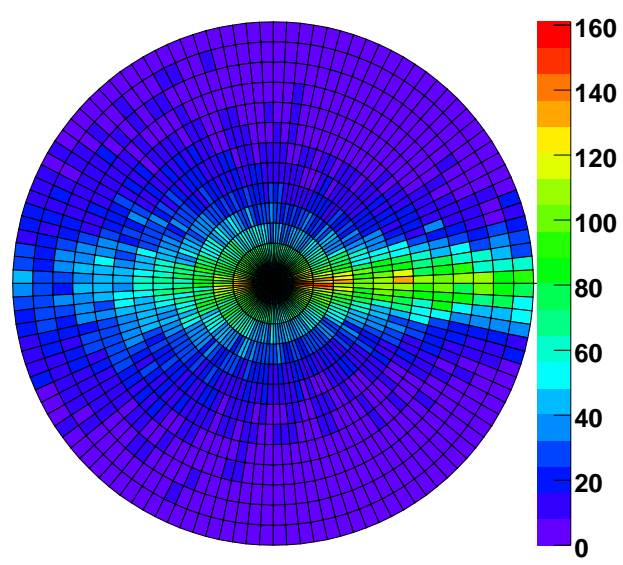

mul: $80-90, \max : 3 \mathrm{GeV}$

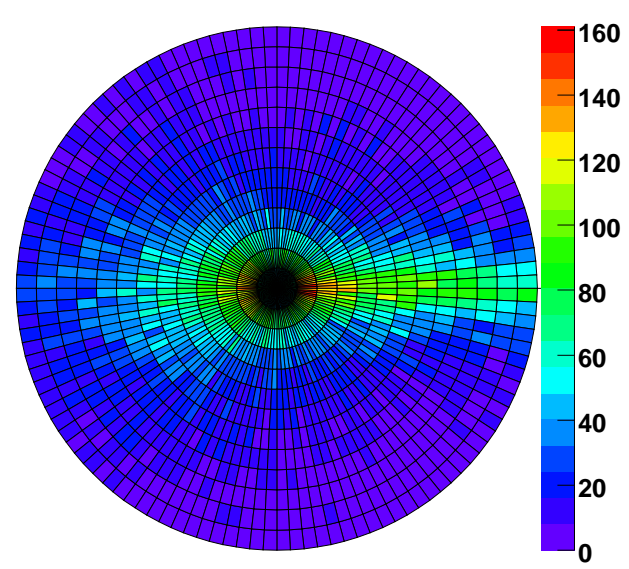

Figure 2: (Colour online.) Charged particle $p_{\mathrm{T}}$ distribution in polar plots. The polar angle is measured relative to the jet axis $\left(\Delta \phi=0\right.$ is at the 3 o'clock position). In the radial direction it is $p_{\mathrm{T}}<3 \mathrm{GeV}$. Colour indicates number of particles. Left panel: intermediate multiplicity events $\left(50 \leq N_{\mathrm{ch}}<60\right)$. Right panel: high multiplicity events $\left(80 \leq N_{\mathrm{ch}}<90\right)$.

jets in the $\Phi-\eta$ space, with the same centre and inner radius as the jet cones and width of $\Delta R$. The surrounding cones grasp the borderline between the jets and the UE, so if there is any kind of correlation between the two regions, it can be seen here. The UE outside the surrounding cones can be considered as free of any influence from the jets.

We have studied Monte Carlo data with our new definitions. The dataset contained $10^{5} \mathrm{p}+\mathrm{p}$ collisions at $\sqrt{s_{N N}}=14 \mathrm{TeV}$, generated by PYTHIA 6. These events where selected after a cut on the transverse momentum-exchange, $Q>100 \mathrm{GeV} / \mathrm{c}$. We applied a simple UA1 jet finder with cone radius 0.7 . The surrounding cone width was considered as $\Delta R=0.1$. On Fig. 2 we display momentum distribution of charged particles, the multiplicity is colour-coded. In the surrounding cones the mean $p_{\mathrm{T}}$ drops drastically [4]. In the whole UE region the characteristic $p_{\mathrm{T}}$ remains under $1 \mathrm{GeV}$. We can also observe the broadening of the away-side jets in high multiplicity events which increases the mean transverse momentum in the UE.

Fig. 2 displays the results obtained from PYTHIA simulation of $p+p$ collisions. The appearance of collective effects could modify substantially the qualitative properties of such histograms. This work was funded by the OTKA grant NK-062044 and the NKTH-OTKA grant H07-C 70464.

\section{References}

[1] A. A. Affolder et al. [CDF Collaboration], Phys. Rev. D 65 (2002) 092002.

[2] S. Catani, Yu. L. Dokshitzer, M. H. Seymour, B. R. Webber, Nucl. Phys. B 406 (1993) 187.

[3] S. D. Ellis, D. E. Soper, Phys. Rev. D 48 (1993) 3160.

[4] A. G. Agócs for the ALICE Collaboration, "Underlying event in p+p collisions with high multiplicities at LHC energies", PoS (HighPT-LHC09) 